\title{
Synthesis of Core/Shell Nanowires Using Doped ZnO Targets
}

\author{
Daisuke NAKAMURA, Kota OKAZAKI, Kazuki KUBO, Koji TSUTA, Mitsuhiro HIGASHIHATA and Tatsuo OKADA \\ Graduate School of Information Science and Electrical Engineering, Kyushu University, \\ 744 Motooka,Nishi-ku,Fukuoka 819-0395, Japan \\ E-mail: dnakamura@ees.kyushu-u.ac.jp
}

\begin{abstract}
$\mathrm{ZnO}$ nanowires have attracted a great attention as building blocks for the optoelectronic devices. For the practical optoelectronic applications based on the $\mathrm{ZnO}$ nanowires, synthesis technique of $\mathrm{ZnO}$ nanowire with layered structures is significantly important in order to achieve a $p-n$ junction, a core/shell structure, and a multiple quantum well structure. We have been succeeded in growing nanowires on the pre-deposited $\mathrm{ZnO}$ film and core/shell structure by a newly developed nanoparticle-assisted pulsed-laser deposition (NAPLD) using multi-target changer. In this paper, recent progresses of synthesis of layer-structured $\mathrm{ZnO}$ nanowires using doped $\mathrm{ZnO}$ target, such as a $\mathrm{Li}-\mathrm{Ni}$ codoped $\mathrm{ZnO}$, are described.
\end{abstract}

DOI:10.2961/jlmn.2012.01.0021

Keywords: ZnO, Pulsed laser deposition, Nanowire, Core/shell structure

\section{Introduction}

Zinc oxide $(\mathrm{ZnO})$ is one of the promising materials in UV optoelectronic applications such as a light-emitting diode (LED) and a laser diode (LD) because of a direct wide band-gap of $3.37 \mathrm{eV}$ at room temperature and a relatively large exciton binding energy of $60 \mathrm{meV}$ [1]. Especially, $\mathrm{ZnO}$ nanowire has attracted a great attention for building blocks of nanodevices such as an UV-LD[2], an UV-LED [3], an UV photodetector [4], and a gas sensor [5], because it has superior crystalline quality, better electrical/optical quality, and large surface area to volume ratio. Furthermore, $\mathrm{ZnO}$ nanowires have no need for a lattice matched substrate for the overgrowth [6]. Those $\mathrm{ZnO}$ nanostructures are able to be synthesized using several methods such as a molecular beam epitaxy [7], sputtering [8], a chemical vapor deposition [9], solution method [10] etc.

For the practical optoelectronic applications based on the $\mathrm{ZnO}$ nanowires, however, three important issues are essentially required: $p$-type doping, growth control and fabrication of layered structures for $p-n$ junction, a coreshell structure, and a multiple quantum well structure, as shown in Fig.1. In our study, we have succeeded in synthesizing various nanostructures, such as nanorods [11], nanowalls [12], the vertically and horizontally aligned $\mathrm{ZnO}$ nanowires [13] by nanoparticle-assisted pulsed-laser deposition (NAPLD) without any catalyst. Furthermore, we have been also succeeded in growing $\mathrm{ZnO}$ nanowires with layered structures, such as a film-wire layered structure and core/shell structure in one NAPLD chamber using the multi-target changer [14]. In this paper, we describe progresses of synthesis and photoluminescence of the layer-structured $\mathrm{ZnO}$ nanowires by NAPLD using doped $\mathrm{ZnO}$ target, such as a $\mathrm{Li}-\mathrm{Ni}$ codoped $\mathrm{ZnO}$, are described.

\section{Experiments}

In the experiment, sintered cylindrical $\mathrm{ZnO}$ source targets were used in synthesizing $\mathrm{ZnO}$ nanowires. A $c$-plane sapphire substrate $(1 \mathrm{~cm} \times 1 \mathrm{~cm})$ was put on a $\mathrm{SiC}$ heater in (a)

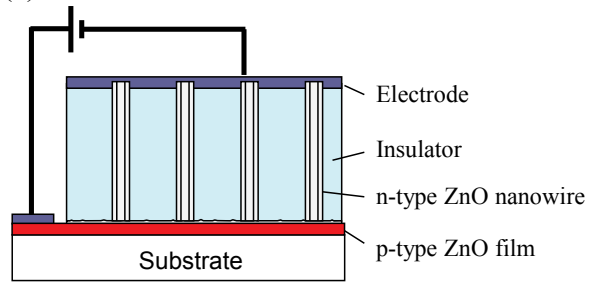

(b)

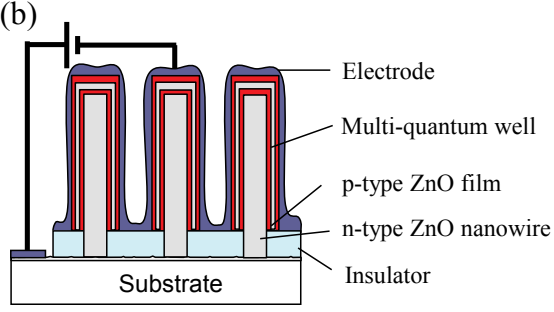

Fig. 1 Schematic of the layer-structured $\mathrm{ZnO}$ nanowires, (a) film-wire structure and (b) core/shell quantum well.

a vacuum chamber and the target-substrate distance was set to $40 \mathrm{~mm}$. The substrate was heated to $400-800{ }^{\circ} \mathrm{C}$ in the vacuum chamber filled with a background gas of argon or oxygen. The $\mathrm{ZnO}$ target was ablated with the third harmonics of a Q-switched Nd:YAG laser at $355 \mathrm{~nm}$ with a repetition rate of $10 \mathrm{~Hz}$ and a fluence of about $1.3 \mathrm{~J} / \mathrm{cm}^{2}$. The morphology of the as-deposited products was analyzed by scanning electron microscopy (SEM). The optical properties of the $\mathrm{ZnO}$ nanowires were investigated by observing the photoluminescence (PL) with a He-Cd laser.

\section{Results and Discussion}

\subsection{Film-Wire Layer-Structured ZnO Nanowires}

We have succeeded in growing the layer-structured $\mathrm{ZnO}$ nanowire consisting of different materials of a pure $\mathrm{ZnO}$ and a Li-Ni codoped $\mathrm{ZnO}$ target [14]. Li-Ni codoped $\mathrm{ZnO}$ is one of the promising targets for $p$-type $\mathrm{ZnO}$ thin film $[15,16]$. $\mathrm{ZnO}$ nanowires were synthesized using the 


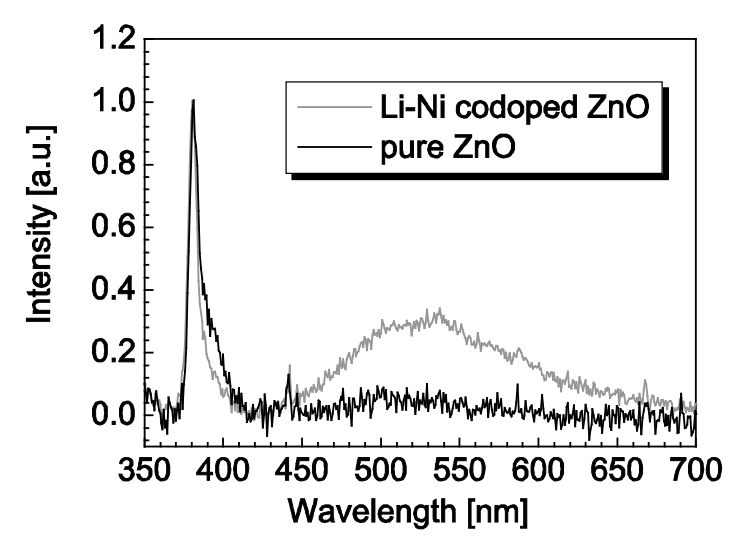

Fig. 2 PL spectrum of the $\mathrm{ZnO}$ nanowires on the $\mathrm{ZnO}$ and the $\mathrm{Li}-\mathrm{Ni}$ codoped $\mathrm{ZnO}$ thin film.

pure $\mathrm{ZnO}$ target after deposition of the Li-Ni codoped $\mathrm{ZnO}$ film. The Li-Ni codoped $\mathrm{ZnO}$ film was deposited on a $c$ plane sapphire substrate temperature of $400^{\circ} \mathrm{C}$ and oxygen gas pressure of 26 mTorr. After deposition of the film for 5 minutes, the nanowires were subsequently synthesized on the film at the temperature of $750{ }^{\circ} \mathrm{C}$ and argon gas of 200 Torr in the same chamber. The growth time of the $\mathrm{ZnO}$ nanowires was $15 \mathrm{~min}$. The vertically-aligned low density $\mathrm{ZnO}$ nanowires with the diameter of 50-100 nm and the length of around $2 \mu \mathrm{m}$ were formed. Most nanowires were grown on hexagonal cone-shape cores. The cores have not been seen in samples without the pre-deposition of the $\mathrm{ZnO}$ film. Besides, most of the cores are formed in the same size using a $\mathrm{Sb}$ doped $\mathrm{ZnO}$ target [17]. Thus the $\mathrm{ZnO}$ film layer probably contributes to the formation of the cores. Although the more detail mechanism should be investigated, an interaction between the buffer film and the depositing nanoparticles occurred.

The optical property of the $\mathrm{ZnO}$ nanowires was characterized with PL spectra measurement at room temperature by a spectrometer (multi-channel analyzer C10027, HAMAMATSU photonics). Fig. 2 shows the PL spectra from the $\mathrm{ZnO}$ nanowires synthesized on the Li-Ni codoped $\mathrm{ZnO}$ and the pure $\mathrm{ZnO}$ thin film. The spectra are normalized at the UV peak for easy comparison. A strong UV peak centered at $380 \mathrm{~nm}$ and a weak broad visible emission with a peak at about $500 \mathrm{~nm}$ were observed from the $\mathrm{ZnO}$ nanowires of the pure $\mathrm{ZnO}$ film. The UV emission band is due to a near-band-edge (NBE) recombination of $\mathrm{ZnO}$, which is the emission of excitons through an electron-hole recombination process [18]. The broad green emission peak of $\mathrm{ZnO}$ is a typical defect-related emission, which is usually attributed to the deep-level oxygen vacancy in the surface and subsurface lattices of $\mathrm{ZnO}$ materials [19]. The intensity of the broad visible emission from the nanowires grown on the Li-Ni codoped $\mathrm{ZnO}$ film is higher than that on the pure $\mathrm{ZnO}$ film. The results show that the Li-Ni thin film might cause an increase of deep-level defects inside $\mathrm{ZnO}$ lattices [20]. However, as an intensity ratio of green emission to NBE from the $\mathrm{ZnO}$ nanowires is less than 0.4 , evident strong UV exciton emission and weaker defect emission show the good crystal quality both of $\mathrm{ZnO}$ nanowires on the pure and Li-Ni codoped films.

\subsection{Core/Shell Structured ZnO Nanowires}

It is important to fabricate layered structure in not only the nanowire axial direction but also the radial direction, because core/shell nanowires are expected to be more efficient in emitting due to low thermal quenching and large surface effect [21]. We have demonstrated the core/shell structured $\mathrm{ZnO}$ nanowieres by NAPLD using pure $\mathrm{ZnO}$ target [14]. After growth of low density $\mathrm{ZnO}$ nanowires at a typical growth condition of the nanowire, the shells were subsequently synthesized under the film deposition condition. It was found that $\mathrm{ZnO}$ shell coats homogeneously the entire nanowire, and thickness of the shell could be controlled by the deposition time.

In this study, core/shell structured $\mathrm{ZnO}$ nanowires consisting of different material layers also could be synthesized using the pure and the $\mathrm{Li}-\mathrm{Ni}$ codoped $\mathrm{ZnO}$ targets. Fig.3(a) shows the SEM image of the pure $\mathrm{ZnO}$ nanowires synthesized after deposition of the $\mathrm{ZnO}$ film. Fig.3(b) shows the SEM image of the $\mathrm{ZnO} / \mathrm{Li}-\mathrm{Ni}$ codoped $\mathrm{ZnO}$ core/shell structued $\mathrm{ZnO}$ nanowires after growth the shell for 10 minutes at the temperature of $400{ }^{\circ} \mathrm{C}$ and oxygen gas pressure of 26 mTorr. Fig. 3(c),(d) shows the $45^{\circ}$ tilted view and top view SEM image of the $\mathrm{ZnO}$ nanowires after growth of the $\mathrm{ZnO}$ shell for 20 minutes. The diameter of the $\mathrm{ZnO}$ nanowires increased uniformly with increasing the deposition time of the shell. On the other hand, the hexagonal cone-shape cores, as seen in Fig.3(a), were buried in the Li-Ni codoped $\mathrm{ZnO}$ film as shown in Fig.3(b),(c). Thus, the incoming $\mathrm{Li}-\mathrm{Ni}$ codoped $\mathrm{ZnO}$ seems to incorporate much more effectively into the $\mathrm{ZnO}$ buffer film layer than the nanowires. These results show the core/shell structure consisting of different $\mathrm{ZnO}$ targets is able to be fabricated by the multi-target changer system in the simple experimental setup.
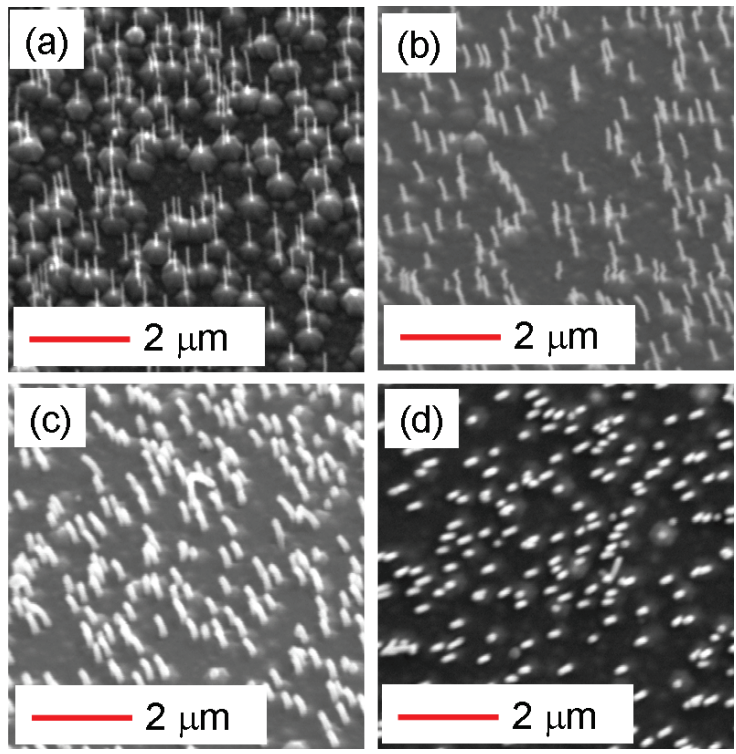

Fig. 3 SEM images of (a) $\mathrm{ZnO}$ nanowires on the film and the $\mathrm{ZnO} / \mathrm{Li}-\mathrm{Ni}$ codoped $\mathrm{ZnO}$ core/shell structued $\mathrm{ZnO}$ nanowires, where the growth time of the $\mathrm{Li}-\mathrm{Ni}$ codoped $\mathrm{ZnO}$ shell layer were (b) $10 \mathrm{~min}$. and 20 min. ((c) $45^{\circ}$ tilted view, (d) top view). 


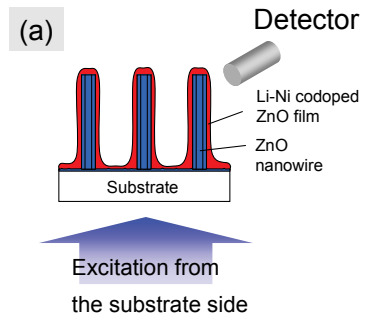

(b) Excitation from the nanowire side the nanowire side

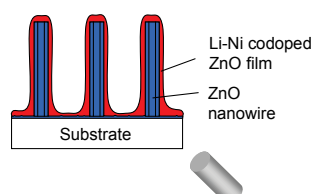

Detector

Fig. 4 Schematic of the PL measurement from the $\mathrm{ZnO} / \mathrm{Li}$ $\mathrm{Ni}$ codoped $\mathrm{ZnO}$ core/shell structued $\mathrm{ZnO}$ nanowires

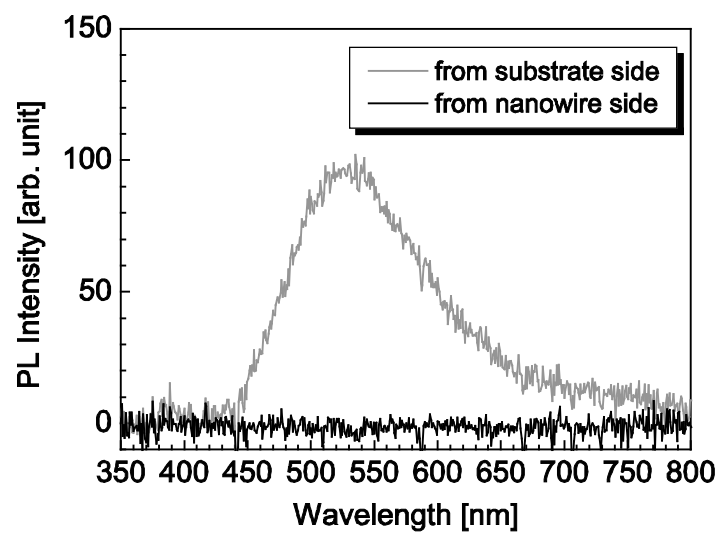

Fig. 5 PL spectrum of the core/shell structured $\mathrm{ZnO}$ nanowire.

The photoluminescence characteristic of the $\mathrm{ZnO} / \mathrm{Li}-\mathrm{Ni}$ codoped $\mathrm{ZnO}$ core/shell structued $\mathrm{ZnO}$ nanowires was also investigated. In the PL measurement, the nanowires were excited from the substrate side or the nanowire side, as shown in Fig.4. Fig.5 shows the PL spectra of the core/shell structured $\mathrm{ZnO}$ nanowires. A visible light emission at green emission band and very low UV emission were observed. On the other hand, no PL spectrum was detected under excitation from the nanowire side, as shown in Fig.5(b). The results indicate the $\mathrm{Li}-\mathrm{Ni}$ codoped $\mathrm{ZnO}$ film absorbs the short wavelength region less than $450 \mathrm{~nm}$. Thus, an intrinsic layer is inevitably required between the $p-n$ junction, which is a PIN ( $p$-type-Intrinsic- $n$-type) diode structure, to fabricate a $\mathrm{ZnO}$-based homo-junction LED using the $\mathrm{Li}-\mathrm{Ni}$ codoped $\mathrm{ZnO}$ as a $p$-type material in order to prevent absorption of the UV emission around the $\mathrm{Li}-\mathrm{Ni}$ codoped $\mathrm{ZnO}$.

\section{Conclusions}

To summarize, we have succeeded in synthesizing layer-structured $\mathrm{ZnO}$ nanowires on $c$-plane sapphire substrates by NAPLD in the single chamber. Vertically-aligned low density $\mathrm{ZnO}$ nanowires were synthesized on the pure and the $\mathrm{Li}-\mathrm{Ni}$ codoped $\mathrm{ZnO}$ film. In addition, core/shell structured $\mathrm{ZnO}$ nanowires consisting of different materials were also fabricated. Furthermore, PL characteristics of the layer-structured $\mathrm{ZnO}$ nanowires were observed under the $\mathrm{He}$ Cd laser excitation.

\section{Acknowledgments}

A part of this study has been financially supported by Special Coordination Funds for Promoting Science and Technology from Japan Science and Technology Agency and a Grant-in Aid for Scientific Research from the Japan Society of Promotion of Science.

\section{References}

[1] Y. Chen, D. M. Bagnall, H-J. Koh, K-T. Park, K. Hiraga, Z. Zhu, and T. Yao: J. Appl. Phys., 84, 3912 (1998).

[2] M. H. Huang, S. Mao, H. Feick, H. Yan, Y. Wu, H. Kind, E. Weber, R. Russo, and P. Yang: Science, 292, 1897 (2001).

[3] M.-C. Jeong, B.-Y. Oh, M.-H. Ham, J.-M. Myounga: Appl. Phys. Lett., 88, 202105 (2006).

[4] Y. Li, F. D. Valle, M. Simonnet, I. Yamada, J.-J. Delaunay: Nanotechnology, 20, 045501 (2009).

[5] Q. Wan, Q. H. Li, Y. J. Chen, T. H. Wang, X. L. He, J. P. Li, and C. L. Lin: Appl. Phys. Lett., 84, 3654 (2004)

[6] M. Willander, L. L. Yang, A. Wadeasa, S. U. Ali, M. H. Asif, Q. X. Zhao and O. Nur: J. Mater. Chem. 19, 1006 (2009).

[7] I.C. Robin, P. Marotel, A.H. EI-Shaer, V. Petukhov, A. Bakin, A. Waag, M. Lafossas, J. Garcia, M. Rosina, A. Ribeaud, S. Brochen, P. Ferret, G. Feuillet: J. Cryst. Growth, 311, 2172 (2009).

[8] Z. Guo, D.X. Zhao, Y. Liu, D. Shen, J. Zhang, B. Liu: Appl. Phys. Lett., 93, 163501 (2008).

[9] J.-J. Wu, S.-C. Liu: Adv. Mater., 14, 215 (2002).

[10] C. H. Ahn, W.S. Han, B. H. Kong, H. K. Cho: Nanotechnology, 20, 015601 (2009).

[11] M. Kawakami, A. B. Hartanto, Y. Nakata, T. Okada: Jpn. J. Appl. Phys., 42, L33 (2003).

[12]B. Q. Cao, T. Matsumoto, M. Matsumoto, M. Higashihata, D. Nakamura, T. Okada: J. Phys. Chem. C, 113, 10975 (2009).

[13]R. Guo, M. Matsumoto, T. Matsumoto, M. Higashihata, D. Nakamura, T. Okada: Appl. Sur. Sci., 255, 9671 (2009).

[14] D. Nakamura, T. Matsumoto, A. Kumeda, K. Toya, K. Okazaki, M. Higashihata, T. Okada: J. Laser Micro/Nanoeng., 6, 23 (2010).

[15]E. S. Kumar, S. Venkatesh, M. S. R. Rao: Appl. Phys. Lett., 96, 232504 (2010).

[16]E. S. Kumar, J. Chatterjee, N. Rama, N. DasGupta, and M. S. R. Rao: Appl. Mater. Interfaces, 3, 1974 (2011).

[17] D. Nakamura, K. Okazaki, I. A. Palani, M. Higashihata, T. Okada, Appl. Phys. A, 103, 959 (2011).

[18]B. Q. Cao, W. P.Cai, H. B. Zeng, Appl. Phys. Lett., 88, 161101 (2006).

[19] S. B. Zhang, S.-H. Wei, and A. Zunger: Phys. Rev. B 63, 075205 (2001).

[20] X. Liu, X. Wu, H. Cao, R. P. H. Chang: J. Appl. Phys., 95, 3141 (2004).

[21]B. Q. Cao, J Zúñiga-Pérez, N. Boukos, C. Czekalla, H. Hilmer, J. Lenzner, A. Travlos, M. Lorenz, M. Grundmann: Nanotechnology, 20, 305701 (2009).

(Received: June 6, 2011, Accepted: January 22, 2012) 